\section{Potentially inappropriate medication in elderly patients with chronic renal disease-is it a problem?}

\author{
Bryony Dean Franklin
}

With an ageing population, safe medication use in older people is becoming even more important. Older people are widely acknowledged to be at higher risk of medication-related problems, and it may be argued that those with chronic renal disease are particularly at risk, as many medications require dose reduction or should be avoided completely in these cases. Jones and Bhandari ${ }^{1}$ report the prevalence of potentially inappropriate prescribing in 100 older people with chronic renal disease admitted to the medical admissions unit of an English teaching hospital. They report that more than half of these patients were prescribed at least one potentially inappropriate medication, and that $13 \%$ of 622 prescribed drugs were potentially inappropriate. The authors indicate that this prevalence is high, and action is therefore required. This raises a number of questions. What does 'potentially inappropriate' actually mean? How should inappropriate medication be identified? Are patients with chronic renal disease more at risk? And, most importantly, what action, if any, is needed?

First, what does 'potentially inappropriate' mean in practice, and why does it matter? Medication that is inappropriate is usually taken to mean medication that is either ineffective or poses an unnecessary risk compared with other options available. However, to explore this in more detail, we also need to consider the next question, concerning methods used for identifying inappropriate medication.

In relation to the second question, two broad approaches to identifying inappropriate medication exist: these are often referred to as 'implicit' and 'explicit' methods. Implicit methods rely on expert judgement and are potentially more subjective with lower inter-rater reliability; however, these methods can take into account wider complexity in relation to the individual patient and thus may have higher validity. In contrast, explicit methods are based on specific criteria for

Correspondence to Professor Bryony Dean Franklin, Department of Pharmacy, Imperial College Healthcare NHS Trust, Charing Cross Hospital, Fulham Palace Road, London W3 9RG, UK;

bryony.deanfranklin@imperial.nhs.uk drugs, diseases and other indicators prepared via expert judgement. They therefore have higher inter-rater reliability, are generally quicker to use, and require less clinical judgement, but do not take into account circumstances surrounding the individual patient. The focus is thus on 'potentially' inappropriate medication (PIM), where a PIM may well be judged to be the best option in an individual patient. Perhaps as a result, the evidence to support a relationship between PIMs and adverse patient outcomes has been inconclusive. ${ }^{23}$ However, because of their relative ease of use, such explicit criteria are widely used, and several sets have been developed with a focus on older people. The Beers criteria, first published in 1991, were the first of these. These were initially developed for frail elderly patients residing in care homes, with subsequent versions modified to include older adults in their own homes. The initial criteria were updated in 1997 and 2003 (the version used by Jones and Bhandari), and then again in 2012. Unusually, Jones and Bhandari supplemented the 2003 Beers criteria with the British National Formulary (BNF) guidance for prescribing in renal impairment, an approach not previously reported.

However, the Beers criteria are by no means the only option, and various other criteria exist. $^{2}{ }^{4}$ Numerous limitations have been identified with Beers, ${ }^{2}$ including the criteria are considered poorly organised, and there is a relatively narrow focus on drugs to be avoided. A major limitation is that the Beers criteria are not generally considered applicable outside the USA, as many of the drugs listed are not available or rarely used elsewhere. Fialova et $a l^{5}$ reported that, overall, half of the medications in the original Beers criteria were not approved in most European countries. The most recent 2012 Beers criteria may fair slightly better, at least in Belgium, where the proportion of applicable criteria increased from $71.2 \%$ for the 2003 criteria to $84.8 \% .^{6}$ An additional limitation is that the Beers criteria are not considered to have been validated in the inpatient setting. ${ }^{2}$ So, what are the alternatives? Levy et $a l^{2}$ identified six sets of criteria developed since
2003 to improve upon the earlier Beers criteria. For example, the Screening Tool of Older Person's Prescriptions (STOPP) criteria are being increasingly used in Europe, and it has been suggested that these be considered the 'European Beers criteria'. 6 As pointed out recently, several studies have shown a greater prevalence of inappropriate prescribing using STOPP compared with Beers, and a link with clinical outcomes has been demonstrated. ${ }^{6}$ Unlike the Beers criteria, the STOPP criteria have also been validated in an inpatient setting. ${ }^{7}$ However, neither Beers nor STOPP take into account the omission of medication that should have been prescribed; instead the Screening Tool to Alert Doctors to Right Treatment specifically addresses prescribing omissions. ${ }^{8}$

Third, are patients with chronic renal disease, as studied by Jones and Bhandari, more at risk? This is a question to which we don't yet know the answer. Jones and Bhandari did identify a higher prevalence of PIMs than in other UK inpatient studies, but, unlike other studies, they used the BNF as well as Beers and unfortunately do not report the proportion of PIMs identified by each. It is therefore impossible to compare their data directly with those of similar studies. They also studied all drugs prescribed on the inpatient chart on admission, including newly prescribed medication as well as that prescribed before admission and then continued in hospital. Previous studies examined only medication prescribed before admission. A further difference is that Jones and Bhandari studied patients aged more than 70 years, whereas previous studies generally focused on the over $65 \mathrm{~s}$. Tools such as Beers and STOPP include very few individual criteria specific to renal function, and other approaches are probably required to answer this question. A recent French study used national renal dose adjustment guidelines and suggested that the risk associated with prescribing in renal disease adds to, rather than being included in, the PIMs identified using Beers. ${ }^{9}$ This is therefore in line with Jones and Bhandari's decision to supplement the Beers criteria with the BNF. However, to add further complexity to this field, there are well-documented differences between different published sources of drug information regarding dosing recommendations for patients with renal disease ${ }^{10}$; the choice of reference source is therefore likely to significantly affect the results obtained.

Finally, what actions are needed? While we cannot directly compare the results of Jones and Bhandari's paper with those in 
the literature, the data certainly suggest that there is room for improvement in prescribing for this population. The prevalence of PIMs in antibiotics, a class of medication likely to be prescribed acutely on hospital admission, was particularly high. This finding suggests that more attention may be needed when prescribing antibiotics in renal impairment in the hospital setting, particularly in older people where a serum creatinine concentration within the reference range can mask a considerable reduction in renal function. The routine reporting of creatinine clearance or estimated glomerular filtration rate is likely to be helpful here, rather than relying on serum creatinine alone. Of note is that the Beers criteria include only one antibiotic (nitrofurantoin), suggesting that Jones and Bhandari identified these PIMs using the BNF.

In summary, PIMs identified using explicit criteria are just that: 'potentially' inappropriate. However, combined with professional clinical judgement, tools to identify PIMs are likely to be useful in identifying patients at risk so that suitable actions can be taken. The paper by Jones and Bhandari suggests that older people with chronic renal disease may be particularly at risk. However, there are various ways to identify PIMs, and clinicians should be familiar with options beyond the Beers criteria, particularly in non-US and inpatient settings.

Acknowledgements The assistance of Matt Reynolds with preparing this paper is gratefully acknowledged.

Contributors BDF conceived and wrote the article.

Competing interests None.

Provenance and peer review Commissioned; internally peer reviewed.

To cite Franklin BD. Postgrad Med J 2013;89:245-246.

Received 25 January 2013

Accepted 25 March 2013

Published Online First 5 April 2013

\section{SLinked}

http://dx.doi.org/10.1136/ postgradmedj-2012-130889

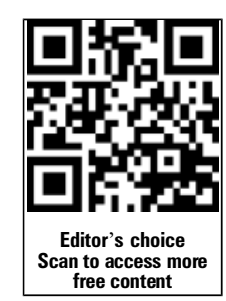

Postgrad Med J 2013;89:245-246. doi:10.1136/postgradmedj-2013-131764

\section{REFERENCES}

1 Jones $S A$, Bhandari $S$. The prevalence of potentially inappropriate medication prescribing in elderly patients with chronic kidney disease. Postgrad Med J 2013;89:247-50.
2 Levy HB, Marcus E-L, Christen C. Beyond the Beers criteria: a comparative overview of explicit criteria. Ann Pharmacother 2010;44: 1968-75.

3 Steinman MA, Rosenthal GE, Landefeld CS, et al. Agreement between drugs-to-avoid criteria and expert assessments of problematic prescribing. Arch Intern Med 2009;169:1326-32.

4 Dimitrow MS, Airaksinen MSA, Kivela S-L, et al. Comparison of prescribing criteria to evaluate the appropriateness of drug treatment in individuals aged 65 and older: a systematic review. J Am Geriatr Soc 2011;59:1521-30.

5 Fialova D, Topinkova E, Gambassi G, et al. Potentially inappropriate medication use among elderly home care patients in Europe. JAMA 2005;293:1348-58.

6 Dalleur O, Boland B, Spinewine A. Updated Beers criteria: greater applicability to Europe? I Am Geriatr Soc 2012;60:2188-9.

7 Gallagher P, O'Mahony D. STOPP (Screening Tool of Older Persons' potentially inappropriate Prescriptions): application to acutely ill elderly patients and comparison with Beers' criteria. Age Ageing 2008;37:673-9.

8 O'Mahoney D, Gallagher P, Ryan C, et al. STOPP and START criteria: a new approach to detecting potentially inappropriate prescribing in old age. Eur Geriatr Med 2010;1:45-51.

9 Breton G, Froissart M, Janus N, et al. Inappropriate drug use and mortality in community-dwelling elderly with impaired kidney function - the Three-City population-based study. Nephrol Dial Transplant 2011;26:2852-9.

10 Vidal L, Shavit M, Fraser A, et al. Systematic comparison of four sources of drug information regarding adjustment of dose for renal function. $B M J$ 2005;331:263-6. 